\title{
PREVALENCE OF CLINICAL SPECTRUM OF INHERITED METABOLIC DISORDERS IN INFANTS AND CHILDREN AT A TERTIARY CARE HOSPITAL IN RAWALPINDI, PAKISTAN
}

\author{
Ayesha Batool, Saeed Zaman*, Ammara Ayub**, Kulsum Bilal ${ }^{* * *}$, Qudratullah Malik*** \\ Combined Military Hospital Lahore/National University of Medical Sciences (NUMS) Pakistan, *Combined Military Hospital Kharian/ \\ National University of Medical Sciences (NUMS) Pakistan, ${ }^{* *}$ Islamic International Medical College, Rawalpindi Pakistan, ${ }^{* * *}$ District \\ Headquarter, Kasur Pakistan, ****Pak Emirates Military Hospital/National University of Medical Sciences (NUMS) Rawalpindi Pakistan
}

\begin{abstract}
Objective: To determine the frequency of a wide range of clinical presentations of inherited metabolic disorders diagnosed in infants and children at a tertiary care hospital in Rawalpindi, Pakistan.

Study Design: Cross sectional study.

Place and Duration of Study: Pak Emirates Military Hospital, Rawalpindi, from Jun 2015 to Jun 2016.

Methodology: Sixty four children diagnosed with metabolic disorders, reporting to the Pak Emirates Military Hospital Rawalpindi, were enrolled in the study. History was taken with special reference to family history, consanguineous marriage, sibling's death and clinical manifestations. Thorough physical examination was done in every patient to find out the clinical signs present. All the data was recorded on a proforma. SPSS-20 version was used to derive the results and $p$-value of $<0.05$ was taken as statistically significant.

Results: In children with metabolic disorders, gastrointestinal manifestation were more significant $78.1 \%(p$-value 0.022). Neurological signs were present in $60.9 \%$ ( $p$-value 0.094$)$ while respiratory manifestations were present in $15.6 \%$ children ( $p$-value 0.251 ). fifty three point one percent were males and $46.9 \%$ were females. The positive history of other affected children in family was significant ( $p$-value 0.015$)$, along with hypoglycaemia ( $p$-value 0.001).

Conclusion: Pallor, failure to thrive, poor feeding, convulsions, lethargy and hypoglycemia were the most frequent clinical manifestations in children with metabolic disorders.
\end{abstract}

Keywords: Inherited metabolic disorders, Glycogen storage disorders, Consanguinity, Convulsions.

This is an Open Access article distributed under the terms of the Creative Commons Attribution License (http://creativecommons.org/licenses/by/4.0), which permits unrestricted use, distribution, and reproduction in any medium, provided the original work is properly cited.

\section{INTRODUCTION}

Inherited metabolic disorders (IMDs) are a group of genetic disorders in which the body cannot properly turn food into energy ${ }^{1}$. These disorders are usually caused by defects in specific proteins (enzymes) that help break down parts of food $^{2}$. The metabolic error is caused due to deficiency of a key enzyme or coenzyme of an intermediary metabolic pathway ${ }^{3}$. IMDs are frequently misdiagnosed or underdiagnosed because of their nonspecific presentations or some neonates with IMDs may die even before screening 4 .

Inborn error of metabolism should be suspected in neonates with metabolic acidosis, alkalosis or with high serum ammonia level5. The term

Correspondence: Dr Ayesha Batool, Child Specialist, Pediatrics Department, $\mathrm{CMH}$ Lahore Pakistan

Received: 07 Feb 2019; revised received: 23 Aug 2019; accepted: 26 Aug 2019 “inborn errors of metabolism' was originally coined by Sir Archibald Garrod at the beginning of the twentieth century. His first publication was on alkaptonuria, he published his most interesting essay entitled 'The Inborn Factors in Disease' 6 .

Knowledge about inherited metabolic disorders is still in its infancy in Pakistan. Although timely diagnosis of metabolic disorders can help in early institution of medical therapy and can help in successful outcome ${ }^{7}$, there are many issues in our country which are related to under diagnosis of these metabolic disorders including lack of awareness about them in general practitioners and paediatricians, their misdiagnosis, variable presentations of symptoms like poor feeding, lethargy and convulsions which resembles many other diseases like sepsis, lack of adequate 
laboratory facilities for diagnostic confirmation in Pakistan and expensive investigations which many families cannot afford.

A great deal of work has been started in Pakistan, including the facilities to diagnose IMDs. Armed Forces Institute of Pathology (AFIP), Rawalpindi has started doing urine organic acid analysis and Plasma amino acid analysis to diagnose various IMDs. We carried out this study with the hope to find out the most common presentations of metabolic disorders in the paediatric population, so that one can know when to have a high index of suspicion, and when to investigate a neonate or child for IMDs as prevention of death or permanent neurological sequels in patients with these disorders is dependent on early diagnosis and establishment of appropriate therapy ${ }^{8}$.

\section{METHODOLOGY}

This was a cross sectional study conducted in paediatrics department, Pak Emirates Military Hospital Rawalpindi after taking permission from hospital ethics committee. Sampling technique used was non-probability consecutive sampling and sample size calculation was done using the WHO sampling calculator. Sixty-four children, who fulfilled the inclusion criteria of having an age range from birth till 12 years and confirmed as having inherited metabolic disorder, falling in any category i.e. disorders of carbohydrate metabolism, disorders of amino acid metabolism, fatty acid oxidation defects, urea cycle defects, organic acidemias, lysosomal storage defects, disorders of electrolyte metabolism etc. were enrolled in the study. All infants and children diagnosed with other congenital infections like TORCH, congenital malaria, tuberculosis, pneumonia, sepsis, neonatal jaundice, hepatitis, connective tissue disorders, malnutrition and infantile leukaemia were excluded. After initial screening laboratory investigations, the diagnosis of specific inherited metabolic disorder was confirmed via respective diagnostic tests to the disorder, including liver biopsy, plasma amino acid analysis by HPLC (High pressure liquid chromatography), urinary organic acid analysis, thyroid function tests, sweat chloride test, urinary porphobilinogen levels, 17-hydroxyprogesterone levels, which were done at the AFIP. History was taken from the parents of the children with especial acknowledgement to the family history, consanguineous marriage, previous sibling's death or other affected children in the family, education of the parents, socioeconomic status, and their knowledge and understanding about the disease. Detailed physical examination of the children was done to find out any visceromegaly, and other clinical signs and symptoms present in the child with respect to gastrointestinal, respiratory and neurological systems, e.g. poor feeding, lethargy, failure to thrive, recurrent vomiting, diarrhea, tachypnoea, respiratory failure, hypoglycaemia, convulsions, hypotonia, delayed developmental milestones, metabolic acidosis and hepatosplenomegaly were noted. This data was collected on a proforma after taking informed consent from parents. SPSS version 20 was applied to derive results, including frequency and percentages of different symptoms found in the children and $p$-value of $<0.05$ was taken as statistically significant.

\section{RESULTS}

Sixty-four children were selected who fulfilled the inclusion criteria, having an age range since birth till 12 years and diagnosed cases of inherited metabolic disorder. Out of them, 53.1\% were males, and $46.9 \%$ were females. Twenty one point nine percent cases had a positive history of other affected children in the family ( $p$-value

Table-I: Frequency of various factors affecting children with inherited metabolic disorders.

\begin{tabular}{l|c|c}
\hline Clinical Manifestations & $\mathbf{n ~ ( \% )}$ & $\boldsymbol{p}$-value \\
\hline Consanguinity & $57(89.1 \%)$ & 0.937 \\
\hline Sibling's death & $22(18.8 \%)$ & 0.945 \\
\hline Hypoglycaemia & $14(21.9 \%)$ & 0.001 \\
\hline Metabolic acidosis & $03(4.7 \%)$ & 0.938 \\
\hline
\end{tabular}

$0.015)$, and the death of siblings was present in $23.4 \%$ cases ( $p$-value 0.945$)$. Consanguinity among parents was present in $89.1 \%$ cases $(p$ value 0.937 ) table-I. 
The most frequent disorder found in our study was the defects in carbohydrate metabolism 25 (39.1\%), followed by amino acid metabolism defects 17 (26.6\%). Four (6.3\%) cases were each of alpha-1 antitrypsin protease deficiency and defects in thyroid hormone synthesis, while $3(4.7 \%)$ cases were of urea cycle defects and defects in electrolyte transport each. There were 2 $(3.1 \%)$ cases, each of organic acidurias and lysosomal storage defects and $1(1.6 \%)$ case of fatty acid oxidation defects, lactic acidemia, mucopolysaccharidosis and heme biosynthesis defect each (figure).

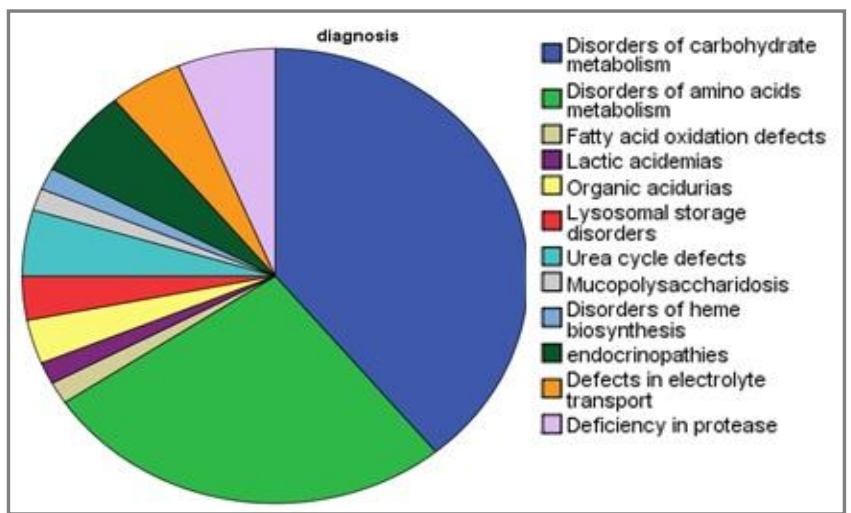

Figure: Frequencies of diverse types of inborn error of metabolism diagnosed in our population.

The gastrointestinal symptoms were noted significant ( $p$-value 0.000 ) in several types of metabolic disorders. The gastrointestinal manifestations were present in $92 \%$ children suffering from the disorders of carbohydrate metabolism and in $76.5 \%$ children having amino acid metabolism defects. While the neurological manifestations were present in $64.7 \%$ children having amino acid metabolism defects and in 52\% children suffering from the disorders of carbohydrate metabolism. Lysosomal storage disorders and organic acidurias presented primarily with gastrointestinal manifestations $(100 \%)$, while both gastrointestinal and neurological complaints were predominant in lactic acidemias, fatty acid oxidation defects, defects in electrolyte transport and in endocrinopathies. Urea cycle defects and disorders of heme biosynthesis were dominated by neurological manifestations.
Among the gastrointestinal symptoms, the most common complaint was pallor in $31(48.4 \%)$ cases, followed by failure to thrive $26(40.6 \%)$, poor feeding 23 (35.9\%), jaundice 22 (34.4\%), recurrent vomiting $7(10.9 \%)$, diarrhoea $3(4.7 \%)$ and dehydration 2 (3.1\%). Among the neurological symptoms, the most frequent presentation in children with IMD was convulsions 31 (48.4\%), followed by lethargy 21 (32.8\%), hypotonia $18(28.1 \%)$, delayed developmental milestones $4(6.3 \%)$ and regression of milestones in 2 $(3.1 \%)$ cases.

Table-II: Frequency of diverse clinical manifestationspresent in infants and children with metabolic disorders.

\begin{tabular}{l|c|c}
\hline Clinical Manifestations & $\mathbf{n ~ ( \% )}$ & $\boldsymbol{p}$-value \\
\hline GI Manifestations: & $51(79.7 \%)$ & $0.022^{*}$ \\
\hline Pallor & $31(48.4 \%)$ & 0.851 \\
\hline Failure to thrive & $26(40.6 \%)$ & 0.124 \\
\hline Poor feeding & $23(35.9 \%)$ & 0.522 \\
\hline Jaundice & $22(34.4 \%)$ & $0.000^{*}$ \\
\hline Recurrent vomiting & $07(10.9 \%)$ & 0.437 \\
\hline Diarrhoea & $03(4.7 \%)$ & 0.407 \\
\hline CNS Manifestations: & $42(65.6 \%)$ & 0.094 \\
\hline Convulsions & $31(48.4 \%)$ & 0.078 \\
\hline Lethargy & $21(32.8 \%)$ & 0.555 \\
\hline Hypotonia & $19(16.2 \%)$ & 0.331 \\
\hline Delayed milestones & $04(3.4 \%)$ & 0.262 \\
\hline $\begin{array}{l}\text { Regression of } \\
\text { milestones }\end{array}$ & $02(1.7 \%)$ & 0.896 \\
\hline $\begin{array}{l}\text { Respiratory } \\
\text { Manifestations }\end{array}$ & $8(12.5 \%)$ & 0.251 \\
\hline Cough & $04(3.4 \%)$ & $0.019^{*}$ \\
\hline Tachypnea & $11(9.4 \%)$ & 0.251 \\
\hline Respiratory failure & $08(6.8 \%)$ & 0.246 \\
\hline
\end{tabular}

${ }^{*} p$-value $<0.05$ is considered significant

Cough 4 (6.3\%) cases, tachypnoea 10 (15.6\%) cases and respiratory failure $8(12.5 \%)$ cases were among the most common respiratory symptoms of metabolic disorders in children. Hypoglycemia was found in $14(21.9 \%)$ cases ( $p$-value 0.001$)$ and metabolic acidosis in $3(4.7 \%)$ cases ( $p$-value 0.938) (table-II).

Among the disorders of carbohydrate metabolism, glycogen storage disorders in 21 (84\%) were the most common disorder found in our 
population, followed by galactosemia in $4(16 \%)$ patients. Among the amino acid metabolism defects, the most common disorder found was non-ketotichyperglycinemia 7 (41.2\%), followed by tyrosinemia 4 (23.5\%), hyperphenylalaninemia $3(17.6 \%)$, and $1(5.9 \%)$ case each of maple syrup urine disease, phenylketonuria and alkaptonuria.

\section{DISCUSSION}

Knowledge about inherited metabolic disorders is still in its infancy in Pakistan. Although timely diagnosis of metabolic disorders can help in early institution of medical therapy and can help in successful outcome, there are many issues in our country which are related to under diagnosis of these metabolic disorders including lack of awareness about them in general practitioners and paediatricians, their misdiagnosis, variable presentations of symptoms like poor feeding, lethargy and convulsions which resembles many other diseases like sepsis, lack of adequate laboratory facilities for diagnostic confirmation in Pakistan and expensive investigations which many families cannot afford.

A great deal of work has been started in Pakistan, including the facilities to diagnose IMDs. Armed forces institute of Pathology (AFIP), Rawalpindi has started doing urine organic acid analysis and Plasma amino acid analysis to diagnose various IMDs. We carried out this study with the hope to find out the most common presentations of metabolic disorders in the paediatric population, so that one can know when to have a high index of suspicion, and when to investigate a neonate or child for IMDs.

The most common clinical presentations found in our population were convulsions and pallor $(48.4 \%)$ followed by failure to thrive $(40.6 \%)$ and poor feeding (35.9\%). We found the metabolic acidosis (4.7\%), hypoglycemia (21.9\%) and history of sibling's death (26.6\%). Our results are comparable to those of another study held in Karnataka, India, where 50 children who presented with neurological and metabolic problems were screened for inborn errors of metabolism and found convulsions (25.7\%) the most common presentation, followed by delayed milestones $(17.9 \%)$, metabolic acidosis $(17.2 \%)$, failure to thrive $(11.7 \%)$, lethargy $(9.4 \%)$, vomiting $(9.4 \%)$ and hypoglycaemia 9 (10.1\%).

History of the sibling's death was found in $26.6 \%$ of cases in our study, which was significantly higher as compared to studies in India, where the death of siblings was found in only $6.2 \%$ cases $^{9}$, while a study in Syria showed unexplained death of siblings in $50 \%$ cases $^{10}$.

Methylmalonic acidemias was the most frequent IMD among organic acidemias found in Syrian populations, and the most frequent symptoms were apnea or respiratory distress (65.7\%) and vomiting $(40 \%)$ cases $^{10}$. Whereas primary lactic acidemia and organic acidemia were the most frequent disorder, followed by amino acidopathiesin a study in India ${ }^{11}$.

In Slovenia, symptomatic patients with suspected IMD were tested and they diagnosed 168 patients with amino acidopathies and organic acidemias, 5 patients with disorders in fatty acid metabolism, 1 patient with a congenital disorder of glycosylation, 42 patients with Fabry disease and 20 patients with Gaucher disease ${ }^{12}$. In Korea, the incidence of IMDs was approximately 1 in 2,235 births ${ }^{13}$, and in Korean population, DUOX2 mutations were found to be a frequent cause of congenital hypothyroidism ${ }^{14}$.

A 13-year retrospective cohort study done in Saudi Arabia showed the incidence of IMDs is 169 in 100,000 births. Of these, 121 patients $(64.7 \%)$ were placed to have small molecule diseases and $66(35.3 \%)$ to take in large molecule diseases. Organic acidemias were the most common small molecule IEMs, while lysosomal storage disorders (LSD) were the most common large molecule diseases and sphingolipidosis were the most common LSD found ${ }^{15}$.

Nevertheless, the studies in Pakistan are limited. A study from Children's Hospital, Lahore, enrolled 239 patients. Agreeing to the study in Lahore, parental consanguinity was found in $97 \%$ of children, which is almost comparable to 
our study in which consanguinity was found in $89.1 \%$ of cases. The most frequent presentation found in that population was a metabolic crisis (32.2\%) followed by neonatal cholestasis (15.5\%) while convulsions (48.4\%) and pallor (48.4\%) were among the most predominant manifestations in our study. The most frequent disorder found in that study was defects in the carbohydrate metabolism (51\%), followed by lipid storage disorders $16(32.7 \%)$ while the most frequent disorder found in our study was defects in the carbohydrate metabolism (39.1\%), followed by amino acid metabolism defects (26.6\%).

Another study at Shifa International Hospital, Islamabad, enrolled 10 patients who underwent diagnostic work-up of IMD and found out that seizures and coma (50\%) were the most common presentations in those children, followed by breathing difficulty (40\%) and vomiting $17(20 \%)$. History of early neonatal death in previous siblings was found in $40 \%$ of patients, while in our study it was found in $18.8 \%$ of cases.

Results conclude that consanguinity is a major contributing factor in our Pakistani population for a specific metabolic disorder, to run in families because most of the IMDs have an autosomal recessive pattern of inheritance ${ }^{18}$. During the period of study, interestingly, it was also found that a considerable number of families were having multiple affected children in the same family because of the high rate of consanguineous marriage in our population.

Besides, by comparing the studies conducted in Pakistan, it can be deducted that disorders of the carbohydrate metabolism defects, amino acid metabolism defects and lipid storage disorders are among the commonest metabolic disorders found in our population.

\section{CONCLUSION}

Inherited metabolic disorders are quite prevalent in our population belonging to all major categories. Most affected children present with predominant gastrointestinal manifestations, including pallor $(48.4 \%)$, failure to thrive $(40.6 \%)$ and poor feeding $(35.9 \%)$ whereas the convulsions $(48.4 \%)$ were the most common neurological manifestation.

\section{CONFLICT OF INTEREST}

This study has no conflict of interest to declare by any author.

\section{REFERENCES}

1. Goetsch AL, Kimelman D, Woodruff TK. Inborn Errors of Metabolism. In: Fertility Preservation and Restoration for Patients with Complex Medical Conditions 2017 (pp.113-139). https://www.axtonbooks.com/shop/product/9783319523156fertility-preservation-and-restoration-for-patients-with-complexmedical-conditions-563131\#attr=1389908,670178,871631,982871.

2. El-Hattab AW. Inborn errors of metabolism. Clinics Perinatology 2015; 42(2): 413-39.

3. Burlina A, Celato A, Burlina AP. Inborn Errors of Metabolism. In Prognosis of Neurological Diseases 2015 (pp. 217-247). Springer Milan.

4. Van Rijt WJ, Koolhaas GD, Bekhof J, Heiner Fokkema MR, de Koning TJ, Visser G, et al. Inborn errors of metabolism that cause sudden infant death: a systematic review with implications for population neonatal screening programmes. Neonatol 2016; 109(4): 297-02.

5. Nyhan WL. When to Suspect Metabolic Disease. In Inherited Metabolic Diseases 2017 (pp. 19-28). Springer Berlin Heidelberg.

6. Gilbert-Barness E, Barness LA, Farrell PM. Metabolic diseases: Foundations of clinical management, genetics, and pathology. IOS Press; 2017 http://ebooks.iospress.nl/book/metabolic-diseases-foundations-of-clinical-management-genetics-andpathology.

7. Ficicioglu C. New tools and approaches to newborn screening: ready to open Pandora's box?. Molecular Case Stud 2017 ; 3(3): a001842.

8. Saudubray JM, Cazorla AG. Clinical Approach to Inborn Errors of Metabolism in Pediatrics. In: Inborn Metabolic Diseases 2016 (pp. 3-70). Springer Berlin Heidelberg.

9. Patil VS, Jailkhani R, Trivedi DJ, Kulkarni SP, Sagare AA, Mudaraddi $\mathrm{R}$, Bargale $\mathrm{A}$. Screening for aminoacidurias and organic acidurias in patients with metabolic or neurological manifestations. Biomedical Research 2012; 23(2): 253-258.

10. Shennar HK, Al-Asmar D, Kaddoura A, Al-Fahoum S. Diagnosis and clinical features of organic acidemias: A hospital-based study in a single center in Damascus, Syria. Qatar Medical J 2015; 2015(1): 9-12.

11. Hampe MH, Panaskar SN, Yadav AA, Ingale PW. Gas chromatography/mass spectrometry-based urine metabolome study in children for inborn errors of metabolism: An Indian experience. ClinBiochem 2017; 50(3): 121-26.

12. Lampret BR, Murko S, Tanšek MŽ, Podkrajšek KT, Debeljak M, Šmon A, et al. Selective Screening for Metabolic Disorders in the Slovenian Pediatric Population. J Medical Biochemistry 2014; 34(1): 58-63.

13. Park KJ, Park S, Lee E, Park JH, Park JH, P. A Population-based genomic study of inherited metabolic diseases detected through newborn screening. Ann Lab Med 2016; 36(6): 561-72.

14. Park KJ, Park HK, Kim YJ, Lee KR, Park JH, Park JH, et al. DUOX2 Mutations Are Frequently Associated With Congenital Hypothyroidism in the Korean Population. Ann Lab Med. 2016; 36(2): 145-53.

15. Alfadhel $\mathrm{M}$, Benmeakel $\mathrm{M}$, Hossain MA, Al Mutairi F, Al Othaim A, Alfares AA, et al. Thirteen-year retrospective review 
of the spectrum of inborn errors of metabolism presenting in a tertiary center in Saudi Arabia. Orphanet J Rare Diseases 2016; 11(1): 126-30.

16. Cheema HA, Malik HS, Parkash A, Fayyaz Z. Spectrum of inherited metabolic disorders in pakistani children presenting at a tertiary care centre. J College Phys Surg Pak 2016; 26(6): 498-02.
17. Choudhry S, Khan M, Rao HA. Etiology and outcome of inborn errors of metabolism. J Pak Med Assoc 2013; 63(9): 1112-16.

18. Zschocke J. Family Issues, Carrier Tests and Prenatal Diagnosis. In Inherited Metabolic Diseases 2017 (pp. 499-503). Springer Berlin Heidelberg. https://www.researchgate.net/publication/ 320427197_Inherited_Metabolic_Diseases_A_Clinical_Approach. 